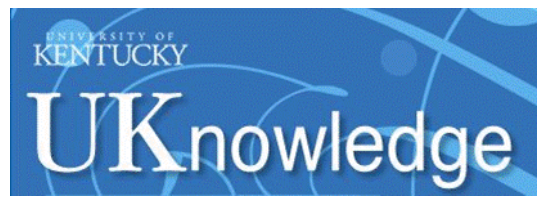

University of Kentucky

UKnowledge

Spinal Cord and Brain Injury Research Center Faculty Publications

Spinal Cord and Brain Injury Research

$1-26-2021$

\title{
Macrophage-Engineered Vesicles for Therapeutic Delivery and Bidirectional Reprogramming of Immune Cell Polarization
}

\author{
Khaga R. Neupane \\ University of Kentucky, krne226@uky.edu \\ J. Robert McCorkle \\ University of Kentucky, rob.mccorkle@uky.edu \\ Timothy J. Kopper \\ University of Kentucky, timothy.kopper@uky.edu \\ Jourdan E. Lakes \\ University of Kentucky, jela237@uky.edu \\ Surya P. Aryal \\ University of Kentucky, Aryalsurya@uky.edu
}

Sellowext thisand fadditionahworkssat: https://uknowledge.uky.edu/scobirc_facpub

Part of the Immunology and Infectious Disease Commons, Medical Physiology Commons, and the Neurology Commons

Right click to open a feedback form in a new tab to let us know how this document benefits you.

\section{Repository Citation}

Neupane, Khaga R.; McCorkle, J. Robert; Kopper, Timothy J.; Lakes, Jourdan E.; Aryal, Surya P.; Abdullah, Masud; Snell, Aaron A.; Gensel, John C.; Kolesar, Jill M.; and Richards, Christopher I., "MacrophageEngineered Vesicles for Therapeutic Delivery and Bidirectional Reprogramming of Immune Cell Polarization" (2021). Spinal Cord and Brain Injury Research Center Faculty Publications. 39. https://uknowledge.uky.edu/scobirc_facpub/39

This Article is brought to you for free and open access by the Spinal Cord and Brain Injury Research at UKnowledge. It has been accepted for inclusion in Spinal Cord and Brain Injury Research Center Faculty Publications by an authorized administrator of UKnowledge. For more information, please contact UKnowledge@lsv.uky.edu. 


\title{
Macrophage-Engineered Vesicles for Therapeutic Delivery and Bidirectional Reprogramming of Immune Cell Polarization
}

\author{
Digital Object Identifier (DOI) \\ https://doi.org/10.1021/acsomega.0c05632 \\ Notes/Citation Information \\ Published in ACS Omega, v. 6, issue 5. \\ (c) 2021 The Authors
}

This is an open access article published under a Creative Commons Non-Commercial No-Derivative Works (CC-BY-NC-ND) Attribution License, which permits copying and redistribution of the article, and creation of adaptations, all for non-commercial purposes.

\section{Authors}

Khaga R. Neupane, J. Robert McCorkle, Timothy J. Kopper, Jourdan E. Lakes, Surya P. Aryal, Masud Abdullah, Aaron A. Snell, John C. Gensel, Jill M. Kolesar, and Christopher I. Richards 


\title{
Macrophage-Engineered Vesicles for Therapeutic Delivery and Bidirectional Reprogramming of Immune Cell Polarization
}

\author{
Khaga R. Neupane, J. Robert McCorkle, Timothy J. Kopper, Jourdan E. Lakes, Surya P. Aryal, \\ Masud Abdullah, Aaron A. Snell, John C. Gensel, Jill Kolesar, and Christopher I. Richards**
}

Cite This: ACS Omega 2021, 6, 3847-3857

Read Online

ACCESS I

Wll Metrics \& More

Article Recommendations

Supporting Information

ABSTRACT: Macrophages, one of the most important phagocytic cells of the immune system, are highly plastic and are known to exhibit diverse roles under different pathological conditions. The ability to repolarize macrophages from pro-inflammatory (M1) to anti-inflammatory (M2) or vice versa offers a promising therapeutic approach for treating various diseases such as traumatic injury and cancer. Herein, it is demonstrated that macrophageengineered vesicles (MEVs) generated by disruption of macrophage cellular membranes can be used as nanocarriers capable of reprogramming macrophages and microglia toward either pro- or

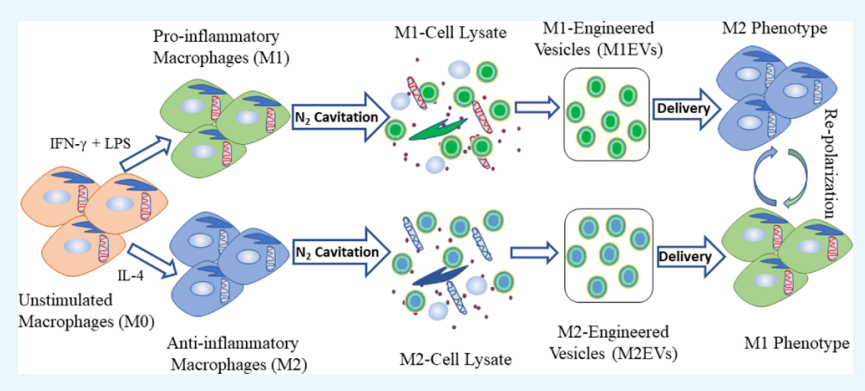
anti-inflammatory phenotypes. MEVs can be produced at high yields and easily loaded with diagnostic molecules or chemotherapeutics and delivered to both macrophages and cancer cells in vitro and in vivo. Overall, MEVs show promise as potential delivery vehicles for both therapeutics and their ability to controllably modulate macrophage/microglia inflammatory phenotypes.

\section{INTRODUCTION}

Macrophages are an essential component of the innate immune system where they play a diverse role. Macrophage function includes clearing waste materials such as cellular debris and participating in tissue repair and remodeling that occurs during wound healing. ${ }^{1}$ They also serve as a defense against bacterial infections and other pathogens largely through phagocytosis. ${ }^{2,3}$ Additionally, they are integral to the initiation of an adaptive immune response through their antigen presenting capabilities. ${ }^{4}$ As a result of this versatile role, macrophages exhibit a range of functional activities which are often driven by stimuli in the surrounding environment. ${ }^{5}$ Macrophages exist in a continuum of polarization states between a pro-inflammatory phenotype, classified as M1, and an anti-inflammatory phenotype, classified as M2. ${ }^{6}$ The polarization state is often mediated by environmental signals such as cytokines, fatty acids, and components from microorganisms such as lipopolysaccharides (LPSs). ${ }^{7,8}$ Pro-inflammatory macrophages are characterized by the production of nitric oxide and the release of high levels of inflammatory cytokines including IL-12, TNF- $\alpha$, and IL-1 $\beta$.1 Anti-inflammatory macrophages secrete cytokines which can dampen the immune response such as IL-10 and IL-4. ${ }^{9}$

The expression of specific macrophage cytokines is implicated in the progression of several disease states. For example, recent studies have shown that macrophages are involved in the progression of cancer, inflammatory diseases, and infectious diseases. ${ }^{10}$ In the tumor microenvironment, macrophages exhibit an anti-inflammatory phenotype and are known as alternatively activated or tumor-associated macrophages (TAMs). ${ }^{11}$ While IFN- $\gamma$ and IL-12 release by proinflammatory macrophages have an anti-angiogenic effect and can block the formation of the new blood vessels in the tumor microenvironment, TAMs suppress production of these cytokines. ${ }^{12-16}$ Factors released by cancer cells in the tumor microenvironment cause TAMs to become tumor-supportive assisting in growth, tissue remodeling, angiogenesis, and metastasis. ${ }^{13,17}$ Tumor progression is further supported by TAMs which produce reduced levels of the major histocompatibility complex (MHC)-II which suppresses the antitumor adaptive immune response. ${ }^{18,19}$ Macrophages also play a critical role in the inflammatory response such as during spinal cord injury (SCI). ${ }^{20}$ As the blood-brain barrier is compromised following SCI, peripheral macrophages rapidly invade the spinal cord and contribute to both pathological and reparative processes. $^{21}$ While pro-inflammatory macrophages contribute to neurodegeneration and tissue loss after SCI, antiinflammatory macrophages contribute to tissue remodeling and axon regeneration. ${ }^{22-24}$ Control of macrophage phenotype

Received: November 18, 2020

Accepted: January 19, 2021

Published: January 26, 2021 
Scheme 1. Schematic Illustrating the Approach of Generating Vesicles from Polarized Bone Marrow-Derived Macrophages ${ }^{a}$

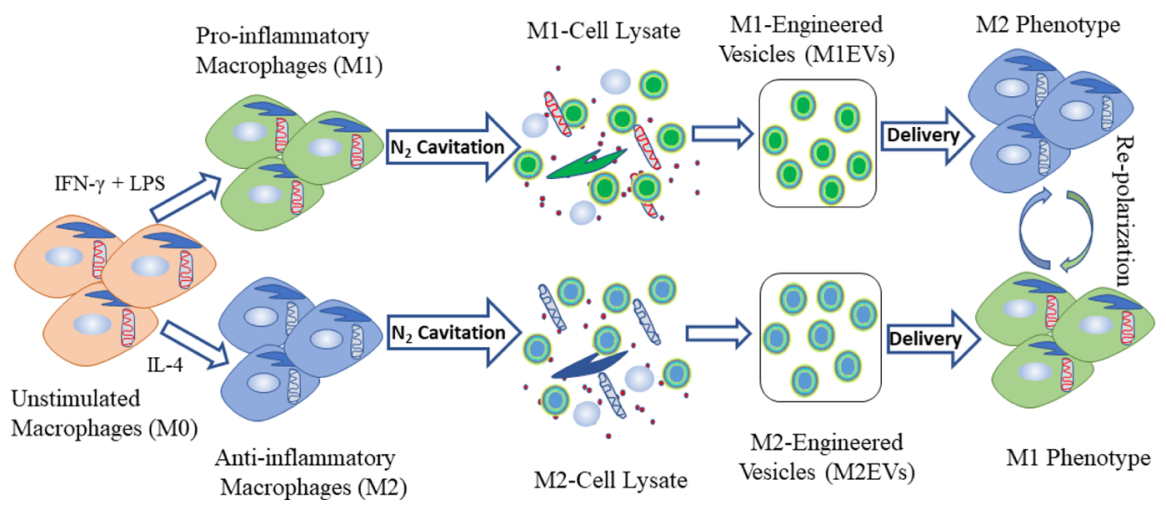

${ }^{a}$ Fully differentiated unstimulated macrophages (M0) are polarized into either pro-inflammatory macrophages (M1) or anti-inflammatory macrophages (M2). Nitrogen cavitation is then used to fragment the cellular membranes of these cells generating M1-engineered vesicles (M1EVs) or M2-engineered vesicles (M2EVs). Vesicles are then separated from cellular fragments by serial centrifugation. These vesicles are then delivered to either unstimulated or polarized macrophages to shift the polarization toward the polarization type of the MEVs.

A

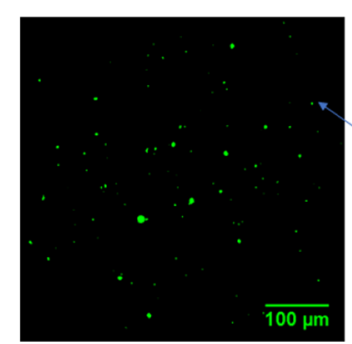

D

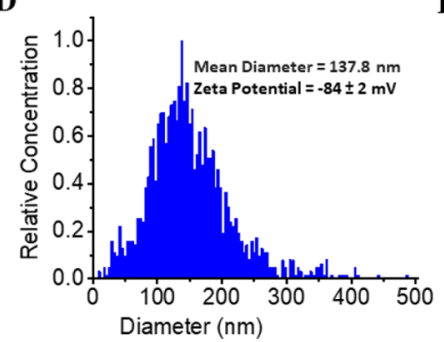

B

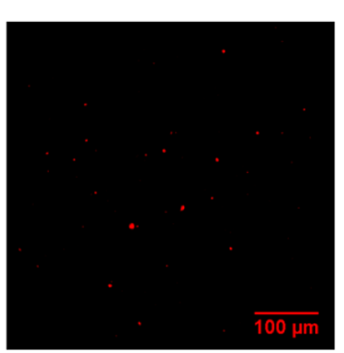

$\mathbf{E}$

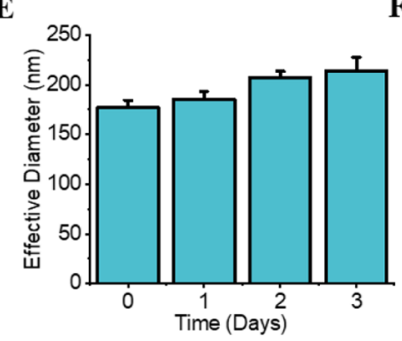

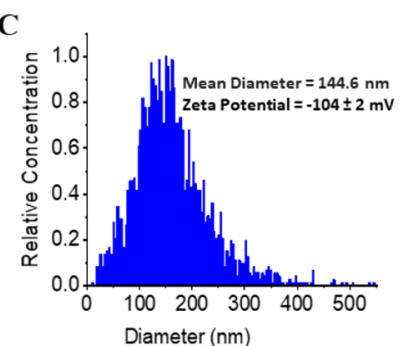

F

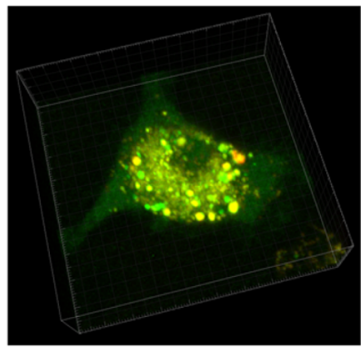

Figure 1. MEV characterization. (A) Fluorescence image of MEVs loaded with a fluorescent dye (fluorescein) during vesicle generation illustrating the principle of encapsulation of cargo by MEVs. (B) Fluorescence image of MEVs labeled with the lipophilic dye, DiI. (C) Size distribution of proinflammatory MEVs (M1EVs) measured by nanoparticle tracking analysis. (D) Size distribution of anti-inflammatory MEVs (M2EVs). The effective diameter of the vesicles generated by nitrogen cavitation was between 100-200 nm. (E) Effective diameter of M1 vesicles in PBS for 3 days measured using dynamic light scattering. (F) 3D confocal image of an M2 macrophage after delivery of fluorescein (interior)-loaded M1EVs labeled with DiI (lipid bilayer), showing clear uptake of vesicles on the surface and inside by macrophages.

through the ability to shift therapeutically between proinflammatory and anti-inflammatory polarizations has been proposed as a potential treatment for diseases such as some types of cancer and traumatic injury. ${ }^{25,26}$ Under different pathological conditions, macrophages exhibit heterogeneity across a continuum of polarization states. The ability to repolarize macrophages from one phenotype to another is a promising technique which might enable alternative forms of treatment for several diseases. For example, repolarizing TAMs toward a pro-inflammatory phenotype is an attractive means to sensitize cancer to immunotherapy. ${ }^{27,28}$ Similarly, repolarizing pro-inflammatory macrophages toward anti-inflammatory phenotypes, thereby reducing the potential neurotoxic effects of M1 macrophages, could be a promising approach for treating SCI and stroke., 24,29

Studies have shown that endogenous extracellular vesicles (EEVs) such as exosomes obtained from immune cells such as macrophages and dendritic cells possess the ability to repolarize TAMs to pro-inflammatory macrophages in the tumor microenvironment. ${ }^{30-32}$ Despite their promise in shifting macrophage phenotype as a therapeutic approach, EEV-based therapies are still challenged by low production yields and difficulties in separating target vesicles from other similarly sized vesicles. ${ }^{33}$ Vesicles artificially generated from cellular membranes have been found to mimic many of the properties of EEVs. ${ }^{28,33-38}$ For example, recent studies demonstrated that vesicles derived from cellular membranes of RAW264.7 cells can stimulate anti-inflammatory macrophages toward a pro-inflammatory phenotype. Studies have also shown that cell-derived vesicles from tumor cells exhibit targeted delivery back to the cell of origin. ${ }^{36}$

In the present study, we generated vesicles from mouse bone marrow-derived macrophages (BMDMs) and demonstrate that we can tune their capability to repolarize macrophages toward 

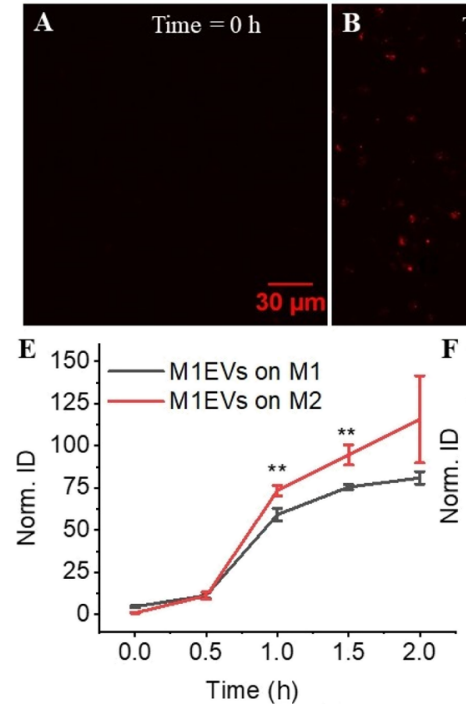
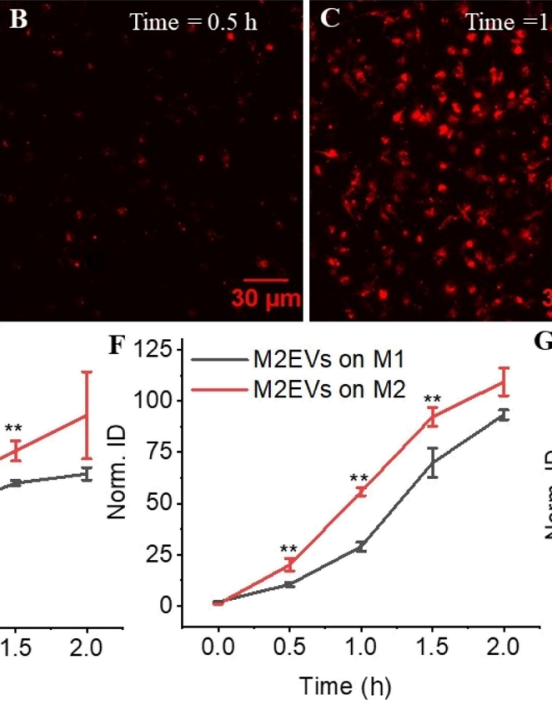
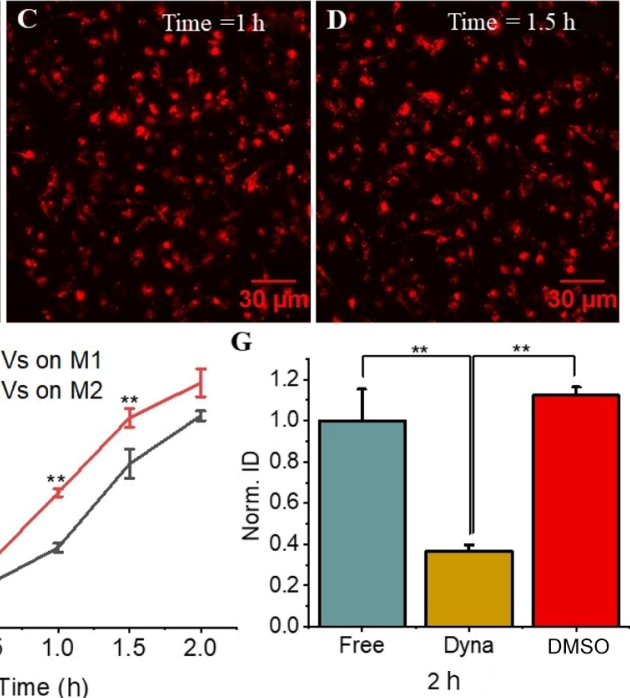

Figure 2. Macrophage targeting specificity. (A-D) Widefield fluorescence images of M2 macrophages showing the time-dependent uptake of DiIlabeled M1EVs by M2 macrophages, scale bar $=30 \mu \mathrm{m}$. (E) Comparison of M1EVs delivered to M1 macrophages (black) vs M1EVs delivered to M2 macrophages (red). (F) Comparison of M2EVs delivered to M1 macrophages (black) vs M2EVs delivered to M2 macrophages (red). (G) Comparison of M2 Macrophages with M1EV delivery (green), M2 macrophages incubated with dynasore ( $80 \mu \mathrm{M})$ for 30 min prior to M1EV addition (gold), and M2 macrophages with M1EV delivery in the presence of DMSO (delivery vehicle) (red). Each data point is the average of five independent replicates $(n=5)$. Norm. ID is the mean integrated density of the image normalized to the mean integrated density value of M2 macrophages before adding vesicles. The data are presented as the mean \pm SEM. $* * p<0.01$ indicates a significant difference in the vesicle uptake by macrophages at respective time points.

either pro- or anti-inflammatory phenotypes. We also characterized these macrophage-engineered vesicles (MEVs) to show that they are similar in size to EEVs and exhibit cell targeting capability for delivery of therapeutics to both cancer cells and macrophages.

\section{RESULTS AND DISCUSSION}

Characterization of MEVs. MEVs are generated through mechanical disruption of the cell membrane into nano-sized fragments which reform into vesicles. Here, we used a prechilled nitrogen decompressor and maintained BMDMs at a pressure of 300 psi for at least $5 \mathrm{~min}$. The sudden release of pressure causes the cell membrane to fragment, and because the phospholipids composing the membrane are amphipathic, the hydrophobic effect drives these fragments to spontaneously form vesicles in aqueous solutions. These vesicles are separated from cellular debris by a series of centrifugation and ultracentrifugation steps as depicted in Scheme 1. Vesicles are generated in the presence of the solution in which the cells were initially suspended, leading to the encapsulation of any hydrophilic therapeutic or other cargo present in the aqueous solution during vesicle generation. Figure 1A shows a fluorescence image of MEVs generated by nitrogen cavitation in the presence of a fluorescein-containing solution. Fluorescein is a fluorescent dye that is soluble in an aqueous medium and is entrapped within the vesicles during their formation. Green punctate regions in the fluorescence image indicate the presence of fluorescein inside the vesicles and the successful loading of cargo during vesicle generation. Similarly, MEVs can be labeled with a lipophilic dye such as DiI. The fluorescence image in Figure $1 \mathrm{~B}$ shows red punctate regions corresponding to DiI incorporation into the vesicle membrane.

To determine the yield of MEVs during nitrogen cavitation, we performed multiple particle tracking to extract both the size distribution of vesicles and their concentration. Particle tracking (Nanosight 300) determines particle size based on diffusion rates and the concentration by counting the number of particles in a defined volume. Vesicles generated from approximately 100 million M1 BMDMs in culture using nitrogen cavitation yielded $5.5 \times 10^{10}$ vesicles (M1EVs). Similarly, 100 million M2 macrophages yielded $6.9 \times 10^{10}$ vesicles (M2EVs). The size distribution of MEVs generated by nitrogen cavitation at 300 psi is primarily between 100-200 $\mathrm{nm}$, which is similar to that of exosomes. ${ }^{39,40}$ The mean diameter of M1EVs was found to be $144.6 \mathrm{~nm}$ (Figure 1C) and that of M2EVs was found to be $137.8 \mathrm{~nm}$ (Figure 1D). We further measured the zeta potential of MEVs suspended in the PBS buffer and found that M1EVs had a zeta potential value of $-104 \pm 2 \mathrm{mV}$ and M2EVs had a zeta potential of $-84 \pm 2 \mathrm{mV}$. A large negative value for the zeta potential indicates the stability of MEVs in aqueous solution. ${ }^{33,41-43}$ These initial characterization studies show that vesicles from BMDMs can be generated with a similar size to exosomes. Additionally, we were able to produce a large number of vesicles from a relatively small volume of tissue culture without the need to wait for long periods of time for the production of EEVs through normal physiological processes.

We next tested the stability of MEVs over time to determine their potential suitability as a drug delivery vehicle where they would be required to circulate within the human body for a period of time before delivery of cargo to a specific site. We tested the stability of MEVs generated by nitrogen cavitation by incubating them in solution for 3 consecutive days. We monitored vesicle size over time to determine the extent of aggregation. The size of MEVs remained relatively constant for the first 2 days, signifying the stability of MEVs over this interval. After $48 \mathrm{~h}$, the stability gradually decreased, as shown by the increase in the size of the vesicles (Figure 1E). Thus, in 


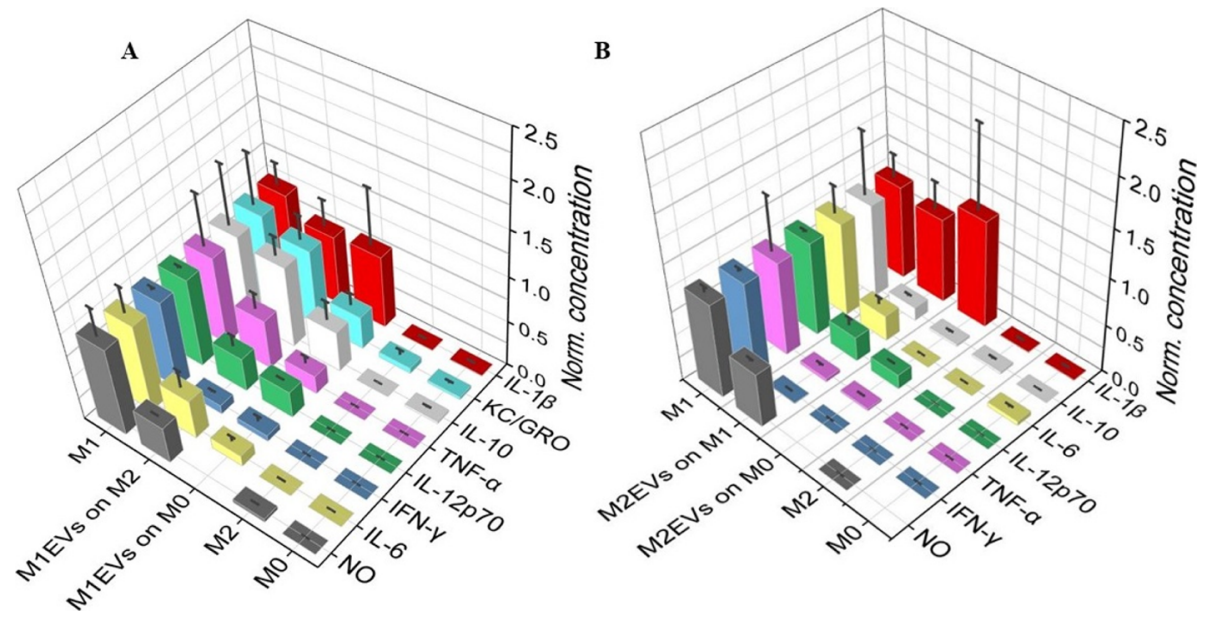

Figure 3. Reprogramming macrophage polarization with MEVs. (A) Measurement of the pro-inflammatory cytokines and nitric oxide (NO) released by M0, M1, and M2 macrophages compared to the production of cytokines released after M1EV delivery to M2 and M0 macrophages. Both M0 and M2 macrophages are polarized toward an M1 phenotype upon interaction with M1EVs in vitro. (B) Quantification of proinflammatory cytokines and NO expression by M0 and M1 macrophages when incubated with M2EVs for $24 \mathrm{~h}$ in vitro. Pro-inflammatory cytokines released by M1 macrophages are significantly reduced upon interaction with M2EVs, which shows that M2EVs are capable of reprogramming M1 macrophages toward an M2 phenotype. Each data point is the average of at least three experiments $(n=3)$. The data are presented as the mean \pm SEM.

addition to their high yields, MEVs are also stable for times compatible with the likely circulation time needed for therapeutic delivery.

MEV Delivery to Macrophages. Previous studies have shown that vesicles generated from cellular membranes can be used as efficient therapeutic delivery vehicles to deliver cargo to the interior of the cell. ${ }^{36}$ In order to investigate the ability of MEVs to deliver cargo into the interior of macrophages, we first generated MEVs from BMDMs stimulated to be M1 (INF- $\gamma+$ LPS) and loaded with fluorescein. The M1EVs were labeled concomitantly with the lipophilic dialkylcarbocyanine fluorescent dye, DiI, which embeds into the lipid bilayer of the vesicles. Both fluorescent labels were separated from the unloaded dye using a size exclusion column. We then incubated BMDMs stimulated with IL-4, to generate M2 cells, with the M1EVs. After incubation with these M2 macrophages, we observed bright fluorescence after $2 \mathrm{~h}$ when imaged with confocal microscopy under both $488 \mathrm{~nm}$ (fluorescein) and $532 \mathrm{~nm}$ (DiI) excitation (Figure S1). M1EVs were evident inside of M2 macrophages, as shown from the fluorescence puncta both inside and on the membrane of macrophages (Figure 1F). At $2 \mathrm{~h}$ after incubation, most vesicles remain intact and isolated on the membrane as well as inside of the cell.

After confirming the delivery of M1EVs onto M2 macrophages, we next performed a set of experiments to determine if vesicles generated from $\mathrm{M} 1$ and M2 BMDMs possess different macrophage targeting capabilities. We generated DiI-labeled vesicles from an equal number of M1 or M2 macrophages. We then determined the efficiency of delivery to M1 and M2 macrophages by measuring the fluorescence signal at various time points over $2 \mathrm{~h}$. We added M1EVs and M2EVs separately to M1 or M2-stimulated BMDMs. Vesicles were then rinsed from the cells, and the cells were subsequently imaged using wide-field microscopy. We found time-dependent uptake of MEVs by macrophages (Figure 2A-D). While both M1EVs and M2EVs were efficiently delivered to $M 1$ and M2 macrophages, M2 macrophages showed a higher uptake of both M1EVs and M2EVs compared to M1 macrophages (Figure 3E,F).

Dynamin activity is an integral component of both endocytosis and phagocytosis. ${ }^{44,45}$ Dynasore, a dynamin inhibitor, has been widely used to study the process of internalization of exosomes from the surface of the macrophage. ${ }^{44,46,47}$ Recent studies showed that the knockdown of dynamin 2 almost completely inhibited the uptake of exosomes by RAW264.7 macrophage-like cells. ${ }^{48}$ Since MEVs mimic exosomes, we next investigated whether they exhibited a similar mechanism of vesicle internalization by macrophages. We compared the uptake of fluorescently labeled M1EVs by M2 BMDMs in the presence and absence of dynasore. Dynasore $(80 \mu \mathrm{M})$ was added to cultured macrophages $20 \mathrm{~min}$ prior to the addition of labeled vesicles. M2 macrophages were left to incubate with M1EVs for $2 \mathrm{~h}$ and subsequently imaged by wide-field microscopy. We found that dynasore had no effect on the cell viability and macrophages looked morphologically similar with and without treatment. We calculated the integrated density of the fluorescence signal to compare the uptake of M1EVs by M2 macrophages. We found that dynasore resulted in $64 \%$ reduction in uptake of vesicles relative to the control (Figure $2 \mathrm{G}$ ). We performed similar vesicle uptake control experiments in the presence of the vehicle, DMSO, at an equal concentration. We found that there was no significant effect of DMSO on the M1EV uptake process by $\mathrm{M} 2$ macrophages relative to the control with no DMSO or dynasore. Macrophages are well-established phagocytotic cells. The loss of cellular uptake with dynamin inhibition coupled with the observation of intact vesicles inside macrophages indicates that macrophages are likely internalizing vesicles via phagocytosis. These results demonstrate that MEVs exhibit similar properties to exosomes and are able to target macrophages.

MEVs Reprogram Macrophage Phenotypes. Previous studies have shown that exosomes generated from M1 or M2 macrophages can be used to differentiate naive macrophages into the corresponding pro- or anti-inflammatory phenotypes. $^{30,49}$ After confirming that MEVs can be delivered to 
macrophages, we tested their ability to differentiate naive (M0) macrophages. M1EVs were generated using nitrogen cavitation from cultured M1 macrophages and then delivered to M0 macrophages to compare cytokine production from M0 macrophages, M1 macrophages, and M0 macrophages incubated with M1EVs. Macrophage-conditioned media (MCM) were extracted from the cell culture of each sample. We performed a meso-scale delivery Sevenplex ELISA that simultaneously tested for seven mouse pro-inflammatory cytokines (IFN- $\gamma$, IL-10, IL-12p70, IL- $1 \beta$, IL-6, KC/GRO, and TNF- $\alpha$ ) in the cell culture supernatant. We observed clear pro-inflammatory markers from M1 macrophages and virtually no measurable levels for most of the cytokines in the M0 culture (Figure 3A). We also found that M1EVs can reprogram M0 macrophages toward an M1 phenotype as evidenced by the increased production of each of the pro-inflammatory cytokines ( $n=3$ /group) from undetectable to $6 \pm 6 \%$ (IFN$\gamma$ ), $45 \pm 2 \%$ (IL-10), $29 \pm 1 \%$ (IL-12p70), $81 \pm 63 \%$ (IL-1 $\beta$ ), $12 \pm 5 \%$ (IL-6), $36 \pm 13 \%(\mathrm{KC} / \mathrm{GRO})$, and $20 \pm 8 \%$ (TNF$\alpha)$ of the average concentration seen for M1 macrophages (Figures 3A and S2). These results verified that M1EVs can stimulate M0 BMDMs toward a pro-inflammatory phenotype. We did not observe a shift toward a pro-inflammatory phenotype when M2MEVs were added to M0 macrophages. Our results reinforce the claim that MEVs exhibit similar properties to exosomes and can be used to polarize naive macrophages.

We next performed a set of experiments to determine the effect of vesicle delivery on macrophages that have already been polarized toward a specific phenotype. We examined the ability of pro-inflammatory vesicles to influence anti-inflammatory macrophages as well as the ability of anti-inflammatory vesicles to influence pro-inflammatory macrophages. To test the capability of MEVs to reprogram already polarized macrophages, we treated cultured M2 BMDMs with M1EVs and compared the cytokine production from M1 macrophages, M2 macrophages, and M2 macrophages exposed to M1EVs. For M2 macrophages that had been treated with M1EVs, we found a significant increase in the production of cytokines $(n=$ 3 /group) from undetectable to $10 \pm 1 \%$ (IFN- $\gamma$ ), $91 \pm 20 \%$ (IL-10), $37 \pm 12 \%$ (IL-12p70), $77 \pm 30 \%$ (IL-1 $\beta$ ), $44 \pm 20 \%$ (IL-6), $85 \pm 27 \%$ (KC/GRO), and $55 \pm 18 \%$ (TNF- $\alpha$ ) of the average concentration seen for M1 macrophages (Figures 3A and S3). We further performed a Griess assay to assess the nitric oxide (NO) presence in MCM collected from M1, M2, and M2 macrophages that were incubated with M1EVs. We found a significant increase in the production of nitric oxide from negligible initial amounts in M2 to $41 \pm 0.4 \%$ of the average concentration seen for M1 macrophages when M2 macrophages were treated with M1EVs. Comparing M2 versus M0 macrophages treated with M1EVs, M1EVs were able to induce a greater increase in pro-inflammatory indicators in M2 macrophages. Control studies showed that MEVs themselves only have marginal amounts of cytokines and they would not be responsible for the amounts seen after the shift (Figure S4). These results indicate that M1EVs can repolarize M2 BMDMs toward a pro-inflammatory M1 phenotype as evidenced by the increase in inflammatory cytokine production.

We also added M2 vesicles to cultured M0 macrophages and compared the cytokine production from M1 macrophages, M0 macrophages, and M0 macrophages incubated with M2EVs (Figure 3B). We found that upon incubation of M0 macrophages with M2EVs, M0 macrophages did not produce most of the pro-inflammatory cytokines, indicating that M2EVs do not induce most of the pro-inflammatory properties in target M0 macrophages (Figure $3 \mathrm{~B}$ ). This indicates that the delivery of vesicles themselves does not simply generate a proinflammatory response that was seen only with M1EV delivery. We further compared the cytokine production from M1 macrophages, M2 macrophages, and M1 macrophages incubated with M2EVs. We observed clear pro-inflammatory markers from M1-macrophages but virtually no levels for most of the pro-inflammatory cytokines in the M2 culture. We further observed a clear decrease in the levels of all the proinflammatory markers for M1 macrophages that were incubated with M2EVs (Figure 3B). M2EVs significantly attenuated cytokine released by M1 macrophages by $99 \%$ (IFN- $\gamma$ ), 85\% (IL-10), 74\% (IL-12p70), 9\% (IL-1 $\beta$ ), 72\% (IL6), $78 \%$ (KC/GRO), and $96 \%$ (TNF- $\alpha$ ) of the average concentration seen for M1 macrophages (Figures 3B and S5). We also observed a significant reduction (49\%) in NO production by M1 macrophages that were incubated with M2EVs compared to the average concentration seen for M1 macrophages. This indicates that M2EVs can reprogram M1 macrophages away from a pro-inflammatory phenotype. This has important implications on the use of MEVs to reprogram macrophage phenotype as part of a therapeutic approach. The phenotype used to generate MEVs appears to dictate their ability to reprogram both naive and already polarized macrophages toward a desired phenotype. The ability to alter macrophage inflammatory properties could be an important therapeutic tool to reprogram anti-inflammatory macrophages to a pro-inflammatory phenotype.

Repolarization of Microglia. Microglia are immune cells present in the central nervous system. ${ }^{50}$ Similar to macrophages, microglia are also polarized to M1 and M2 phenotypes and play pro- and anti-inflammatory roles, respectively. ${ }^{51}$ To determine if macrophage-derived vesicles are able to reprogram microglia phenotypes, we delivered vesicles derived from macrophages to primary microglia cells in culture. We induced M2 microglia polarization using IL-4. M1EVs generated from bone marrow-derived M1 (LPS + INF- $\gamma$ ) macrophages were then added to cultured M2 microglia to compare the cytokine production from M1 microglia, M2 microglia, and M2 microglia incubated with M1EVs. We observed clear proinflammatory markers from M1 microglia and virtually no measurable levels for most of the cytokines in the M2 microglia culture. We also observed an increase in the levels of all the pro-inflammatory markers for M2 microglia that were incubated with M1EVs (Figure S6). The ability of M1EVs to reprogram M2-polarized microglia toward a pro-inflammatory (M1) phenotype in a controlled fashion suggests that we can reprogram both macrophage and microglia inflammatory properties by the delivery of vesicles that are targeted to specific cell types. Furthermore, similar to macrophage exosomes, MEVs can deliver the corresponding signals to unstimulated macrophages and differentiate them into specific phenotypes. This has implications for therapeutic approaches where the goal is to either initiate or suppress a proinflammatory response.

The ability of MEVs to reprogram immune cells is likely due to membrane-bound proteins on the surface of the vesicle. As they are derived from parent immune cells, MEVs carry a wide range of transmembrane proteins, membrane-bound cytokines, and other cell signaling endogenous ligands. These proteins 
can interact with membrane receptors on the target cell initiating signaling cascades that lead to repolarization.

Macrophage-Induced Neurotoxicity. Classically activated M1 macrophages, stimulated with LPS + IFN- $\gamma$, are neurotoxic and contribute to neuronal degeneration by releasing high levels of specific pro-inflammatory cytokines and oxidative metabolites such as nitric oxides. ${ }^{9,52}$ Proinflammatory cytokines such as TNF- $\alpha$, IFN- $\gamma$, IL-12, and IL- 6 have been found to be involved in neuronal death. ${ }^{53-55}$ Alternatively activated, M2 macrophages do not induce cell death but rather help the repair process by releasing growth factors and anti-inflammatory cytokines. ${ }^{22,23}$ Recent studies showed that azithromycin (AZM), a frequently used macrolide antibiotic, also possesses the ability to reduce macrophagemediated neurotoxicity by altering macrophage phenotype from pro-inflammatory to anti-inflammatory. ${ }^{9,56}$ We sought to determine if MEV-induced reprogramming of M1 macrophages toward an M2 phenotype could moderate neurotoxicity in a similar fashion to AZM. ${ }^{9}$ We used LPS + IFN- $\gamma$ to stimulate an M1 macrophage phenotype and IL-4 to stimulate an M2 phenotype. We generated vesicles from M2 macrophages and then exposed M1 macrophages to M2 MEVs, which reduces the production of pro-inflammatory cytokines (Figure 3B). We collected the supernatant from M1 macrophages and M1 macrophages that had been exposed to M2EVs for $24 \mathrm{~h}$. Media from both conditions were used to separately treat differentiated Neuro-2A (N2a) cells. N2a cells are a mouse neural crest-derived cell line which possess the ability to differentiate into cells with neuron-like characteristics. We found that media from M1 macrophages resulted in a $40 \%$ reduction in neuron viability relative to the control media (Figure 4A) ( $n=5 /$ group). We further found that media collected from M1 macrophages that had been exposed to M2EVs for $24 \mathrm{~h}$ resulted in no significant reduction in neuron viability relative to the control (Figure 4A). This is likely due to the significant reduction of pro-inflammatory cytokines by M1 macrophages upon incubation with M2EVs (comparable to AZM-treated M1 cells, Figure 4B), and the corresponding
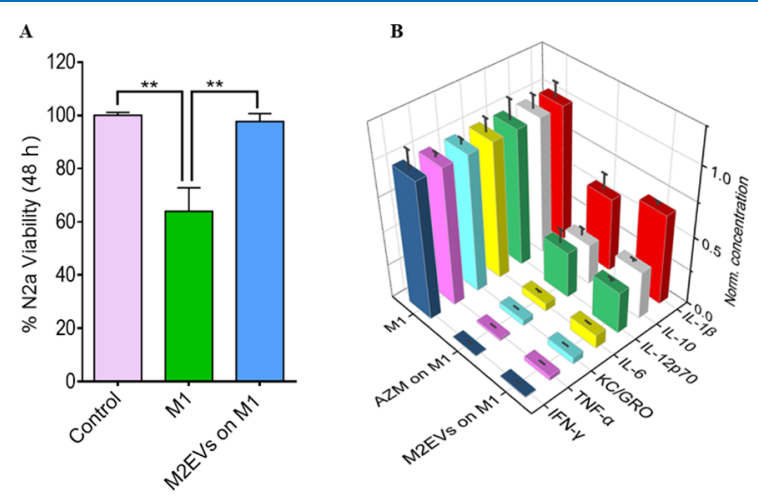

Figure 4. Macrophage-mediated neurotoxicity. (A) The effect of macrophage-conditioned media on the viability of differentiated N2a cells was determined using a cell viability assay for control cells with growth media (pink), for the supernatant from M1 macrophage culture (green), and for the supernatant from M1 macrophage culture after treatment with M2EVs (blue). (B) Comparative study of the ability of M2EVs and AZM in solution $(10 \mu \mathrm{M})$ to reprogram M1 macrophages toward an M2 phenotype. Each data point is the average of five independent replicates $(n=5)$. ** $p<0.01$ indicates that the results are statistically significant. The data are presented as the mean \pm SEM. increase in neuron viability suggests that pro-inflammatory cytokines released by M1 macrophages play a major role in the cytotoxicity of $\mathrm{N} 2 \mathrm{a}$ cells. These results also indicate that reprogramming M1 macrophages toward an anti-inflammatory phenotype using M2EVs is comparable to an immunomodulatory pharmacological agent and reduces the cytotoxicity normally observed with pro-inflammatory macrophages.

MEVs for Therapeutic Delivery. Previous studies have shown that vesicles generated from A549 (lung carcinoma) cells can target as well as deliver chemotherapeutics to the same cell type from which they were generated. ${ }^{36}$ There is some concern about the use of cancer cell-derived vesicles for drug delivery because of the potential for these vesicles to be cleared by the body's immune system and that these vesicles might increase the metastatic potential. We tested MEVs to determine if they had similar targeting and therapeutic delivery features as were previously observed for cancer cell vesicles. MEVs lack any cancer characteristics and would not increase the metastatic potential. We first performed an experiment to determine the targeting ability of MEVs for A549 cells. We generated vesicles from macrophages and labeled them with DiI. We then determined the efficiency of delivery of MEVs by measuring the fluorescence signal at time points over $4 \mathrm{~h}$. We observed an increase in the fluorescence intensity over time resulting from an uptake of MEVs by the A549 cells. The uptake of MEVs by A549 cells suggests that MEVs can serve as a potential drug delivery vehicle in the delivery of chemotherapeutics (Figure S7). We next determined if MEVs could be loaded with cisplatin and delivered to cancer cells while maintaining the efficacy of the therapeutic. We also compared the specificity of cisplatin delivery onto A549 cells using M0EVs, M1EVs, and M2EVs (Figure 5A). We found that

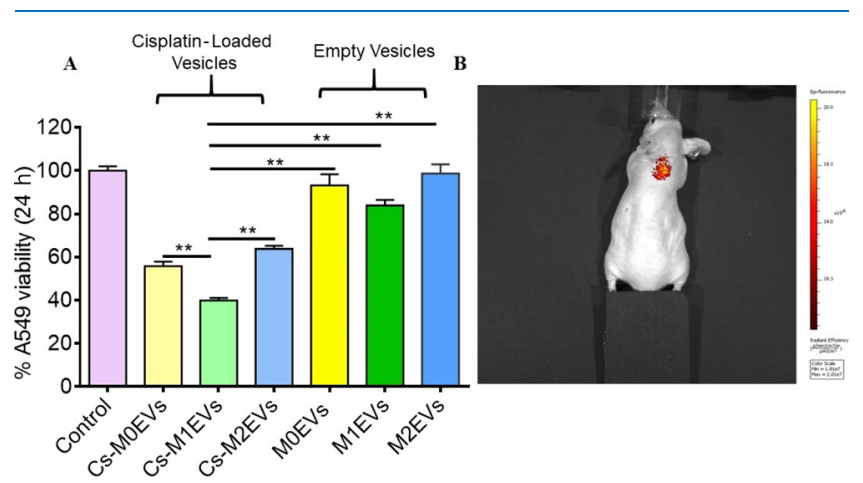

Figure 5. MEVs as biological nanocarriers. (A) Comparison of targeting specificity of cisplatin-loaded M0, M1, and M2-engineered vesicles to A549 cells. Each data point is the average of five independent replicates $(n=5)$. ANOVA with post hoc Tukey's HSD was used to test the significance of the results. $* * p<0.01$ indicates that the results are statistically significant. The data are presented as the mean \pm SEM. (B) Mice bearing A549 xenografts were injected with $\mathrm{DiR}$ labelled M1EVs, demonstrating that M1EVs can reach the tumor of the mice.

empty M0EVs and M2EVs had no significant effect on A549 cell proliferation. However, M1EVs resulted in $10 \%$ A549 cell death in $24 \mathrm{~h}$. We further generated cisplatin-loaded MOEVs (Cs-M0EVs), M1EVs (Cs-M1EVs), and M2EVs (Cs-M2EVs) from an equal number of M0, M1, and M2 macrophages. Previous studies have shown that vesicles generated using nitrogen cavitation can efficiently encapsulate chemotherapeutics and are stable for 2 days. ${ }^{36}$ Therapeutic-loaded MEVs were 
then delivered to cancer cells to determine cytotoxicity. Cisplatin-loaded M0 and M2 vesicles resulted in 45 and $40 \%$ cell death, respectively, at $24 \mathrm{~h}$. However, cisplatin-loaded M1 vesicles resulted in a $60 \%$ A549 cell death in $24 \mathrm{~h}$ (Figure 5A). This is a clear indication that cisplatin-loaded M1 MEVs are more efficient in killing cancer cells compared to cisplatinloaded M0 and cisplatin-loaded M2 macrophages.

In Vivo Delivery of MEVs to Tumor Xenografts. To determine if MEVs exhibited similar targeting features in vivo as observed in cell culture, we generated vesicles from M1 macrophages and labeled them with a membrane dye, DiR. The free dye was separated from MEVs using PD Miniprep columns. We found that M1EVs targeted tumor xenografts (subcutaneous injection A549 cells) implanted in immunecompromised athymic nude $(\mathrm{nu} / \mathrm{nu})$ mice. After the tumor xenograft reached at least $100 \mathrm{~mm}^{3}$, we injected $2 \times 10^{10}$ vesicles through the tail vein of each of the three different mice. We used an IVIS whole animal imager for in vivo imaging. Imaging was done at 48 and $72 \mathrm{~h}$ post injection of labeled vesicles. DiR alone when injected into the mice as a control showed nonspecific accumulation. We observed clear delivery of the labeled vesicles to the tumor xenograft at $72 \mathrm{~h}$ post injection of labeled vesicles (Figure 5B). These results verify that M1EVs can specifically target the tumor tissue in vivo.

\section{CONCLUSIONS}

In conclusion, BMDMs can be used to engineer nano-sized vesicles with high yield using nitrogen cavitation. These vesicles can be loaded with various cargo during their generation and can be used as drug delivery vehicles both in vitro and in vivo. In addition, MEVs when interacting with the macrophage itself possess the ability to reprogram macrophages and microglia into specific inflammatory phenotypes that dictate the macrophage function (e.g., neurotoxicity and tumor migration). This shows the potential for MEVs as a novel and versatile therapeutic to target and reprogram macrophages.

\section{EXPERIMENTAL SECTION}

Animals. We used 2-5-month-old wild-type C57BL/6 mice to extract bone marrow cells. Animals were properly accommodated in IVC cages by providing enough food and water. All experiments were performed following the guidelines of the National Institute of Health and were approved by the Institutional Animal Care and Use Committee at the University of Kentucky.

Cell Culture. BMDMs were isolated from both tibias and femurs of wild-type mice at 2-5 months of age as previously reported. ${ }^{23,57}$ Briefly, mice were first anesthetized and then killed by cervical dislocation. After removing femurs and tibias from the carcass, the bone marrow was extracted using a 10 $\mathrm{mL}$ syringe loaded with Roswell Park i Institute (RPMI) Medium into a $50 \mathrm{~mL}$ centrifuge tube. The bone marrow in media was then triturated with an 18-gauge needle until a single cell suspension was obtained, followed by centrifugation at $1,200 \times g$ for $5 \mathrm{~min}$. The supernatant was carefully removed, and cells were resuspended in $4 \mathrm{~mL}$ of RBC lysis buffer $(0.15$ $\mathrm{M} \mathrm{NH}_{4} \mathrm{Cl}, 10 \mathrm{mM} \mathrm{KHCO}$, and $0.1 \mathrm{mM} \mathrm{Na}_{4}$ EDTA), followed by swirling by hand for $3 \mathrm{~min}$. $6 \mathrm{~mL}$ of RPMI media was then added, followed by centrifugation at $1200 \times g$ for $5 \mathrm{~min}$. The supernatant was aspirated off, and the cells were resuspended in differentiation media (RPMI supplemented with $10 \%$ fetal bovine serum (FBS), 1\% penicillin/streptomycin (PS), 1\% (4(2-hydroxyethyl)-1-piperazineethanesulfonic acid), $0.001 \% \beta$ mercaptoethanol, $1 \%$ glutamine, and $20 \%$ supernatant from sL929 cells) and plated in T-175 cell culture flasks in differentiation media. sL929 cell lines were maintained in RPMI media supplemented with $10 \%$ FBS, $1 \%$ PS, and $1 \%$ glutamine. The supernatant from sL929 cells contains the macrophage-colony stimulating factor (MCSF), which is essential for differentiating bone marrow cells into macrophages. Differentiation media were replaced on days 2, 4, and 6 , and the cells were replated on day 7 at a cell density of $1 \times$ $10^{6}$ cells $/ \mathrm{mL}$ in replating media [Dulbecco's modified Eagle's medium (DMEM) supplemented with $10 \%$ FBS, $1 \%$ glutamine, and $1 \%$ PS). On day 8 , cells were stimulated to M1 [LPS (20 ng/mL; Invivogen) + IFN- $\gamma(20 \mathrm{ng} / \mathrm{mL}$; eBioscience $)$ ] or M2 [IL-4 (20 ng/mL); eBioscience] macrophages, while the unstimulated macrophages from day 7 were termed M0 macrophages. For cytokine analysis, the supernatant from stimulated cells, MCM, was collected after 24 $\mathrm{h}$. Vesicles were added after $12 \mathrm{~h}$ of stimulation, and the supernatant was collected after $24 \mathrm{~h}$ of vesicle addition to M1 or M2 macrophages. The MCM obtained were collected into Eppendorf tubes and stored at $-80{ }^{\circ} \mathrm{C}$ until the analysis was done.

Primary cultures of microglia were prepared from postnatal P2 to P4 pups from C57BL/6 mice. Briefly, pups were decapitated, and brains were kept in Petri dishes filled with icecold Hank's Balanced Salt Solution $\left(\mathrm{Ca}^{2+}, \mathrm{Mg}^{2+}, \mathrm{NaHCO}_{3}\right.$, and phenol red). Brains were dissected, and the hippocampal region was extracted for microglia isolation and culture. The tissues were then minced, and the cell suspension was made. The cell suspension was treated with $2.5 \%$ trypsin (quality biological), incubated, and finally resuspended in the astrocyte culture media containing DMEM with 10\% FBS and 1\% PS. Cells were incubated at a density of 2 million on a poly-Llysine-coated T75 flask containing astrocyte culture media. Cell culture media were changed every 3 days until the flask was confluent with cells. Microglia were detached from astrocytes and oligodendrocytes by shaking the flasks for 30 min at a speed of $180 \mathrm{rpm}$.

The mouse neuroblastoma cell line (also known as Neuro-2a or $\mathrm{N} 2 \mathrm{a}$ ) was maintained in the $\mathrm{N} 2 \mathrm{a}$ cell culture medium composed of 44\% DMEM, 45\% OPTI-MEM reduced-serum medium, $10 \%$ FBS, and $1 \%$ PS. A total of 40,000 N2a cells were plated in each well of a 96-well plate in N2a media supplemented with $20 \mu \mathrm{M}$ retinoic acid (Sigma-Aldrich) and allowed to differentiate for $24 \mathrm{~h}$. Retinoic acid helped N2a cells to differentiate into cells with neuron-like properties. ${ }^{58}$ On day 1 , the differentiation media were exchanged for $100 \mu \mathrm{L}$ of various $\mathrm{MCM}$ in $20 \mu \mathrm{M}$ retinoic acid and two controls with and without $20 \mu \mathrm{M}$ retinoic acid. Cells were further incubated for $48 \mathrm{~h}$, and the neurotoxicity of MCM was evaluated using an alamar blue cell proliferation assay.

Human lung cancer (A549) cells were maintained in the A549 cell culture medium composed of $89 \%$ DMEM, $10 \%$ FBS, and $1 \%$ PS. A total of 40,000 A549 cells were plated in each well of a 96-well plate and left to incubate for $12 \mathrm{~h}$ at 37 ${ }^{\circ} \mathrm{C}$. After $12 \mathrm{~h}$, the old growth media were removed carefully, being sure not to disturb the cells, and were exchanged with A549 cell media containing cisplatin-loaded macrophage (M0, $\mathrm{M} 1$, and M2)-engineered vesicles or empty (M0, M1, and M2) vesicles. After $24 \mathrm{~h}$ of incubation at $37^{\circ} \mathrm{C}$, the media were 
aspirated off and $100 \mu \mathrm{L}$ of Optimem was added, followed by $20 \mu \mathrm{L}$ of alamar blue for the cell viability assay.

Cell Viability Assay. For cytotoxicity assays, the cell media from each well of a 96-well plate were exchanged for $100 \mu \mathrm{L}$ of Optimem (Invitrogen), followed by the addition of $20 \mu \mathrm{L}$ of alamar blue. Cells were then incubated for 35-45 min until a uniform purple coloration was developed. The resulting fluorescence was measured using a Tecan 96-well plate reader equipped with an excitation filer set to $535 \mathrm{~nm}$ and the emission filter set to $595 \mathrm{~nm}$. All measurements were done in quintuplicate (five different wells), and at least three independent experiments were carried out.

MEV Isolation. Completely differentiated macrophages from day 8 were used to generate MEVs. The macrophage cell media were aspirated off from the flask containing macrophages, and the cells were first washed with PBS. $3 \mathrm{~mL}$ of PBS was further added to each flask, and cells were detached by scraping them, followed by resuspension in PBS. The cell suspension from all flasks was first collected into a $50 \mathrm{~mL}$ tube, and the total number of cells was counted using a hematocytometer. The cell slurry collected in the previous step was then centrifuged at $1200 \mathrm{rpm}$ at $4{ }^{\circ} \mathrm{C}$ for $5 \mathrm{~min}$, and the obtained pellets were resuspended in $10 \mathrm{~mL}$ of PBS supplemented with the protease inhibitor. To fragment the cellular membrane and generate the vesicles, cells were then subjected to a pressure of 300 psi for $5 \mathrm{~min}$ in a prechilled nitrogen gas decompressor (Parr Instruments Company, IL, USA) on ice. The pressure was rapidly released to generate fragmentation resulting in vesicles. The fragmented cell mixture including vesicles was centrifuged at $4000 \times g$ for 10 min at $4{ }^{\circ} \mathrm{C}$. The pellet obtained was discarded, but the supernatant was centrifuged at $10,000 \times g$ for $20 \mathrm{~min}$ at $4{ }^{\circ} \mathrm{C}$. The supernatant was again subjected to ultracentrifugation at $100,000 \times \mathrm{g}$ for $60 \mathrm{~min}$ at $4{ }^{\circ} \mathrm{C}$ to pellet the remaining nanovesicles. The pellet was washed five times with PBS before being resuspended in $500 \mu \mathrm{L}$ of the PBS buffer.

MEV Characterization. MEVs were generated by nitrogen cavitation, followed by a series of centrifugation steps as discussed above. The mean diameter, concentration, and zeta potential of MEVs were determined via nanoparticle tracking analysis (NTA) using a Nanosight 300 and a ZetaView PMX120. Similarly, MEV stability was determined using dynamic light scattering (DLS). A ZetaPALS potential Analyzer (Brookhaven Instruments) was used to obtain the DLS measurements.

MEV Labeling. Cells were detached from the flask and counted and resuspended in $9.9 \mathrm{~mL}$ of PBS. $100 \mu \mathrm{L}$ of 100 $\mathrm{mM}$ fluorescein was added to the cell suspension so that the final concentration of fluorescein becomes $1 \mathrm{mM}$ in the cell suspension. The cell solution was fragmented using nitrogen cavitation, and the vesicle pellet was obtained. The pellet was then washed with PBS to remove any unincorporated fluorescein inside the vesicle. Vesicles were then resuspended in $1 \mathrm{~mL}$ of $\mathrm{PBS}$ and transferred to a clean ultracentrifuge (UCF) tube where the vesicle suspension was diluted to $4 \mathrm{~mL}$ in PBS. For the complete removal of the free dye, the diluted vesicle suspension was recentrifuged at $100,000 \times \mathrm{g}$ for $60 \mathrm{~min}$ at $4{ }^{\circ} \mathrm{C}$. The supernatant from centrifugation was discarded, and the pellet was washed with $1 \mathrm{~mL}$ of the PBS buffer. $500 \mu \mathrm{L}$ of PBS was added to the UCF tube, and the pellet was resuspended by pipetting several times. DiI was then added to the vesicle resuspension such that the final concentration of the dye becomes $2 \mu \mathrm{M}$ and left to incubate for $30 \mathrm{~min}$ at $37^{\circ} \mathrm{C}$.
DiI is a lipophilic dye which gets incorporated into the lipid bilayer of the vesicle. The free dye molecules were separated from the fluorescently labeled vesicles using a size exclusion spin column (PD MidiTrap column). The column was equilibrated first by running $15 \mathrm{~mL}$ of PBS through the column and the column was centrifuged at $1000 \mathrm{~g}$ for 2 min to remove any remaining PBS from the column. Then, $500 \mu \mathrm{L}$ of the vesicle solution was added carefully onto the center of the column from the top and centrifuged at $1000 \mathrm{~g}$ for $2 \mathrm{~min}$ to obtain DiI-labeled vesicles loaded with fluorescein.

MEV Imaging. DiI or fluorescein-labeled vesicles were generated as discussed previously and deposited onto a glass bottom dish before imaging them using fluorescence microscopy. DiI-labeled vesicles were imaged using a $532 \mathrm{~nm}$ laser of a $1.9 \mathrm{~mW}$ power with a gain of 990 and an exposure time of $200 \mathrm{~ms}$. Similarly, fluorescein-loaded vesicles were imaged using a $488 \mathrm{~nm}$ laser of a $0.8 \mathrm{~mW}$ power with a gain of 990 and an exposure time of $200 \mathrm{~ms}$.

Confocal Imaging. A Nikon A1R laser scanning confocal microscope equipped with a $60 \times$ oil objective was used for confocal imaging of macrophages that had taken up dyelabeled vesicles. Thus, the obtained images were analyzed with Nikon image processing software. ${ }^{59}$

MEV Uptake. 100 million M1 and 110 million M2 macrophages were used to prepare M1EVs and M2EVs, respectively, for the study of MEV uptake by M1 or M2 macrophages. MEVs were generated and labeled with DiI as mentioned previously. From total $500 \mu \mathrm{L}$ of each vesicle suspension, $50 \mu \mathrm{L}$ of DiI-labeled vesicles was then added separately to each glass bottom dish containing 90,000 M1 or M2 macrophages. Imaging was done at $0.5,1,1.5$, and $2 \mathrm{~h}$ using a fluorescence microscope equipped with a $20 \times$ objective with an exposure time of $32 \mathrm{~ms}$. The macrophage media with fluorescently labeled vesicles were first removed, and the cells were washed twice with $1 \mathrm{~mL}$ of L-15 prior to the addition of 1 $\mathrm{mL}$ of $\mathrm{L}-15$ to the cells for imaging.

Cisplatin-Loaded MEVs. 100 million M0, M1, or M2 cells were used to generate macrophage-derived, cisplatin-loaded vesicles and deliver them to A549 cells. Macrophage media were first aspirated off, and $3 \mathrm{~mL}$ of PBS was added to each flask prior to scraping them. The cell solution was collected into a $50 \mathrm{~mL}$ centrifuge tube, and the number of cells was determined using a hematocytometer. The cell solution was pelleted at $2000 \times g$ for $2 \mathrm{~min}$ at $4{ }^{\circ} \mathrm{C}$. The supernatant was discarded, and cells were resuspended in $8 \mathrm{~mL}$ of $8.33 \mathrm{mM}$ cisplatin solution made in PBS with 1 tablet of the protease inhibitor. The cell solution was nitrogen-cavitated using a prechilled nitrogen decompressor on ice at 300 psi for $5 \mathrm{~min}$. The cell lysate obtained was centrifuged at $4000 \times g$ for $10 \mathrm{~min}$ at $4{ }^{\circ} \mathrm{C}$. The pellet thus obtained was discarded, and the obtained supernatant was centrifuged at $10,000 \times \mathrm{g}$ for $20 \mathrm{~min}$ at $4{ }^{\circ} \mathrm{C}$. The supernatant obtained was again subjected to ultracentrifugation at $100,000 \times g$ for $60 \mathrm{~min}$ at $4{ }^{\circ} \mathrm{C}$ to collect the pellet containing cisplatin-loaded nanovesicles. This final pellet was first washed with $1 \mathrm{~mL}$ of PBS twice and resuspended in $750 \mu \mathrm{L}$ of PBS. Empty vesicles were generated using the same procedure discussed above but in the absence of cisplatin.

Cisplatin Concentration in MEVs. The concentration of cisplatin loaded in vesicles was determined using inductively coupled plasma-optical emission spectrometry (ICP-OES). ${ }^{36}$ Cisplatin-loaded MEVs were first treated with $1 \%$ Triton X100 to dissolve the lipid bilayer, followed by $70 \%$ nitric acid 
treatment to release platinum from cisplatin. The resulting solution was further incubated on a heat block at $60^{\circ} \mathrm{C}$ for $2 \mathrm{~h}$, followed by dilution to $5 \mathrm{~mL}$, such that the final nitric acid concentration was $10 \%$ for analysis using ICP-OES. A standard curve using platinum standards in $10 \%$ nitric acid solution was used to determine the concentration. Ytterbium was used as an internal standard to compensate for the internal drift of the instrument. We have previously shown that vesicles generated by nitrogen cavitation are stable with no apparent cisplatin leakage for $72 \mathrm{~h}^{36}$

Cytokine Analysis. MEVs were generated as described before. M1EVs were generated from 100 million M1 macrophages and resuspended in $500 \mu \mathrm{L}$ of PBS. The number of vesicles present in the resuspension was determined using NTA. $5.49 \times 10^{9}$ M1EVs were added into each well of a 24well plate containing 1 million M0 and M2 macrophages in $950 \mu \mathrm{L}$ of replating media. The plate was left to incubate at 37 ${ }^{\circ} \mathrm{C}$ for $24 \mathrm{~h}$. After $24 \mathrm{~h}$ of incubation, MCM were collected in an Eppendorf tube $(1 \mathrm{~mL})$ and later used for pro-inflammatory cytokine analysis. M2EVs were generated as before using M2 macrophages. $7.6 \times 10^{9} \mathrm{M} 2 \mathrm{EVs}$ were added to each well containing M0 and M1 macrophages. The plate was left to incubate at $37{ }^{\circ} \mathrm{C}$ for $24 \mathrm{~h}$ before collecting the media for cytokine analysis. We performed a mouse pro-inflammatory sevenplex assay following the manufacturer's protocol. Briefly, $25 \mu \mathrm{L}$ of calibrators and MCM were added to each well of a capture antibody-precoated MSD well plate. The plate was then allowed to incubate for an hour, and the detection antibody was added into each well of the MSD. After vigorously shaking the plate for an hour, it was then washed with $0.5 \%$ tween PBS. The Read buffer was finally added to each well and analyzed on the MESO SECTOR imager from Meso Scale Discovery. Standard curves were obtained by fitting the electrochemiluminescence signal from calibrators using Meso Scale Delivery Workbench analysis software.

In Vivo Delivery. A549 cells $\left(1 \times 10^{6}\right)$ were injected subcutaneously into the interscapular region of 6-week-old athymic nude mice. The mice were monitored until palpable xenograft tumors developed greater than $200 \mathrm{~mm}^{3}$. M1EVs were generated using 100 million M1 macrophages by the procedure mentioned above. A NanoSight 300 multiple particle tracking system was used to determine the mean diameter and the concentration of MEVs. M1EVs were then labeled with the DiR near-infrared fluorescent dye. Briefly, 1 $\mu \mathrm{L}$ of $1 \mathrm{mM}$ DiR was added to $199 \mu \mathrm{L}$ of the vesicle resuspension so that the final concentration of $\mathrm{DiR}$ in the vesicle resuspension was $5 \mu \mathrm{M}$. DiR-labeled vesicles were separated from free DiR using a size exclusion PD MidiTrap column equilibrated with PBS. $100 \mu \mathrm{L}$ of DiR-labeled M1EVs was then injected into the lateral tail vein of tumor-bearing mice. Isoflurane gas was used to anesthetize mice for imaging $72 \mathrm{~h}$ post injection using an IVIS Spectrum In Vivo Imaging System (PerkinElmer) controlled with LivingImage software (PerkinElmer). Epifluorescence images were obtained using $710 \mathrm{~nm}$ excitation and $760 \mathrm{~nm}$ emission filters, $\mathrm{f} /$ stop number 4 and binning factor 4 , with a 35 s exposure.

Statistical Analysis. Statistical analyses were performed using Origin 2018. All data were expressed as the mean \pm standard error of the mean (SEM). At least three independent biological replicate experiments were performed for each condition $(n \geq 3)$. The two-sample $t$-test or ANOVA with post hoc Tukey's HSD test was done when appropriate and results were considered statistically significant at p-values less than or equal to 0.01 .

\section{ASSOCIATED CONTENT}

\section{SI Supporting Information}

The Supporting Information is available free of charge at https://pubs.acs.org/doi/10.1021/acsomega.0c05632.

Vesicle delivery and cytokine production (PDF)

\section{AUTHOR INFORMATION}

\section{Corresponding Author}

Christopher I. Richards - Department of Chemistry, University of Kentucky, Lexington, Kentucky 40506, United States; (1) orcid.org/0000-0003-0019-1989;

Email: chris.richards@uky.edu

\section{Authors}

Khaga R. Neupane - Department of Chemistry, University of Kentucky, Lexington, Kentucky 40506, United States

J. Robert McCorkle - Department of Pharmacy Practice and Science, College of Pharmacy, University of Kentucky, Lexington, Kentucky 40508, United States

Timothy J. Kopper - Spinal Cord and Brain Injury Research Center, Department of Physiology University of Kentucky, Lexington, Kentucky 40536, United States

Jourdan E. Lakes - Department of Chemistry, University of Kentucky, Lexington, Kentucky 40506, United States

Surya P. Aryal - Department of Chemistry, University of Kentucky, Lexington, Kentucky 40506, United States

Masud Abdullah - Department of Chemistry, University of Kentucky, Lexington, Kentucky 40506, United States

Aaron A. Snell - Department of Chemistry, University of Kentucky, Lexington, Kentucky 40506, United States

John C. Gensel - Spinal Cord and Brain Injury Research Center, Department of Physiology University of Kentucky, Lexington, Kentucky 40536, United States

Jill Kolesar - Department of Pharmacy Practice and Science, College of Pharmacy, University of Kentucky, Lexington, Kentucky 40508, United States

Complete contact information is available at:

https://pubs.acs.org/10.1021/acsomega.0c05632

\section{Author Contributions}

K.R.N and C.I.R wrote the manuscript. K.R.N, T.J.K, J.L, S. P.A, M.A, and A.A.S performed the experiments. C.I.R, J.K, and J.C.G designed the experiments.

\section{Notes}

The authors declare no competing financial interest.

\section{ACKNOWLEDGMENTS}

We thank the Light Microscopy Core at the University of Kentucky for confocal microscopy experiments. We also thank Dr. Jennifer S. Moylan, Director at the Biomarker Analysis Lab at the University of Kentucky for providing access to the Meso Sector for MSD analysis. Support for this work was provided by the Kentucky Pediatric Cancer Research Trust Fund (PON2 728 2000002500).

\section{REFERENCES}

(1) Mantovani, A.; Biswas, S. K.; Galdiero, M. R.; Sica, A.; Locati, M. Macrophage plasticity and polarization in tissue repair and remodelling. J. Pathol. 2013, 229, 176-185. 
(2) Hirayama, D.; Iida, T.; Nakase, H. The Phagocytic Function of Macrophage-Enforcing Innate Immunity and Tissue Homeostasis. Int. J. Mol. Sci. 2017, 19, 92.

(3) Duque, G. A.; Descoteaux, A. Macrophage cytokines: involvement in immunity and infectious diseases. Front. Immunol. 2014, 5, 491.

(4) Elhelu, M. The role of macrophages in immunology. J. Natl. Med. Assoc. 1983, 75, 314-317.

(5) Stout, R. D.; Suttles, J. Functional plasticity of macrophages: reversible adaptation to changing microenvironments. J. Leukocyte Biol. 2004, 76, 509-513.

(6) Mills, C. D.; Kincaid, K.; Alt, J. M.; Heilman, M. J.; Hill, A. M. M-1/M-2 macrophages and the Th1/Th2 paradigm. J. Immunol. 2000, 164, 6166-6173.

(7) Biswas, S. K.; Mantovani, A. Macrophage plasticity and interaction with lymphocyte subsets: cancer as a paradigm. Nat. Immunol. 2010, 11, 889.

(8) Sica, A.; Mantovani, A. Macrophage plasticity and polarization: in vivo veritas. J. Clin. Invest. 2012, 122, 787-795.

(9) Zhang, B.; Bailey, W. M.; Kopper, T. J.; Orr, M. B.; Feola, D. J.; Gensel, J. C. Azithromycin Drives Alternative Macrophage Activation and Improves Recovery and Tissue Sparing in Contusion Spinal Cord Injury. J. Neuroinflammation 2015, 12, 218.

(10) Cheng, H.; Wang, Z.; Fu, L.; Xu, T. Macrophage Polarization in the Development and Progression of Ovarian Cancers: An Overview. Front. Oncol. 2019, 9, 421.

(11) Ramanathan, S.; Jagannathan, N. Tumor associated macrophage: a review on the phenotypes, traits and functions. Iran. J. Cancer Prev. 2014, 7, 1-8.

(12) Sun, L.; He, C.; Nair, L.; Yeung, J.; Egwuagu, C. E. Interleukin 12 (IL-12) family cytokines: Role in immune pathogenesis and treatment of CNS autoimmune disease. Cytokine 2015, 75, 249-255.

(13) Lin, Y.; Xu, J.; Lan, H. Tumor-associated macrophages in tumor metastasis: biological roles and clinical therapeutic applications. J. Hematol. Oncol. 2019, 12, 76.

(14) Anestakis, D.; Petanidis, S.; Kalyvas, S.; Nday, C.; Tsave, O.; Kioseoglou, E.; Salifoglou, A. Mechanisms and applications of interleukins in Cancer Immunotherapy. Int. J. Mol. Sci. 2015, 16, 1691-1710.

(15) Sgadari, C.; Angiolillo, A.; Tosato, G. Inhibition of Angiogenesis by Interleukin-12 Is Mediated by the Interferon-Inducible Protein 10. Blood 1996, 87, 3877-3882.

(16) Sorensen, E. W.; Gerber, S. A.; Frelinger, J. G.; Lord, E. M. IL12 suppresses vascular endothelial growth factor receptor 3 expression on tumor vessels by two distinct IFN- $\gamma$-dependent mechanisms. J. Immunol. 2010, 184, 1858-1866.

(17) Mantovani, A.; Marchesi, F.; Malesci, A.; Laghi, L.; Allavena, P. Tumour-associated macrophages as treatment targets in oncology. Nat. Rev. Clin. Oncol. 2017, 14, 399-416.

(18) Chang, Y.-C.; Chen, T.-C.; Lee, C.-T.; Yang, C.-Y.; Wang, H.W.; Wang, C.-C.; Hsieh, S.-L. Epigenetic control of MHC class II expression in tumor-associated macrophages by decoy receptor 3 . Blood 2008, 111, 5054-5063.

(19) Baumgart, M.; Moos, V.; Schuhbauer, D.; Müller, B. Differential expression of major histocompatibility complex class II genes on murine macrophages associated with $\mathrm{T}$ cell cytokine profile and protective/suppressive effects. Proc. Natl. Acad. Sci. U.S.A. 1998, 95, 6936-6940.

(20) David, S.; Kroner, A. Repertoire of microglial and macrophage responses after spinal cord injury. Nat. Rev. Neurosci. 2011, 12, 388.

(21) Greenhalgh, A. D.; David, S. Differences in the Phagocytic Response of Microglia and Peripheral Macrophages after Spinal Cord Injury and Its Effects on Cell Death. J. Neurosci. 2014, 34, 63166322.

(22) Kigerl, K. A.; Gensel, J. C.; Ankeny, D. P.; Alexander, J. K.; Donnelly, D. J.; Popovich, P. G. Identification of Two Distinct Macrophage Subsets with Divergent Effects Causing either Neurotoxicity or Regeneration in the Injured Mouse Spinal Cord. J. Neurosci. 2009, 29, 13435-13444.
(23) Gensel, J. C.; Nakamura, S.; Guan, Z.; van Rooijen, N.; Ankeny, D. P.; Popovich, P. G. Macrophages Promote Axon Regeneration with Concurrent Neurotoxicity. J. Neurosci. 2009, 29, 3956-3968.

(24) Gensel, J. C.; Zhang, B. Macrophage activation and its role in repair and pathology after spinal cord injury. Brain Res. 2015, 1619, $1-11$.

(25) Brown, J. M.; Recht, L.; Strober, S. The Promise of Targeting Macrophages in Cancer Therapy. Clin. Cancer Res. 2017, 23, 32413250.

(26) Novak, M. L.; Weinheimer-Haus, E. M.; Koh, T. J. Macrophage activation and skeletal muscle healing following traumatic injury. $J$. Pathol. 2014, 232, 344-355.

(27) Tariq, M.; Zhang, J.; Liang, G.; Ding, L.; He, Q.; Yang, B. Macrophage Polarization: Anti-Cancer Strategies to Target TumorAssociated Macrophage in Breast Cancer. J. Cell. Biochem. 2017, 118, 2484-2501.

(28) Choo, Y. W.; Kang, M.; Kim, H. Y.; Han, J.; Kang, S.; Lee, J.-R.; Jeong, G.-J.; Kwon, S. P.; Song, S. Y.; Go, S.; Jung, M.; Hong, J.; Kim, B.-S. M1 Macrophage-Derived Nanovesicles Potentiate the Anticancer Efficacy of Immune Checkpoint Inhibitors. ACS Nano 2018, 12, 8977-8993.

(29) Gensel, J. C.; Kopper, T. J.; Zhang, B.; Orr, M. B.; Bailey, W. M. Predictive screening of M1 and M2 macrophages reveals the immunomodulatory effectiveness of post spinal cord injury azithromycin treatment. Sci. Rep. 2017, 7, 40144.

(30) Cheng, L.; Wang, Y.; Huang, L. Exosomes from M1-Polarized Macrophages Potentiate the Cancer Vaccine by Creating a Proinflammatory Microenvironment in the Lymph Node. Mol. Ther. 2017, 25, 1665-1675.

(31) Wu, T.; Qi, Y.; Zhang, D.; Song, Q.; Yang, C.; Hu, X.; Bao, Y.; Zhao, Y.; Zhang, Z. Bone Marrow Dendritic Cells Derived Microvesicles for Combinational Immunochemotherapy against Tumor. Adv. Funct. Mater. 2017, 27, 1703191.

(32) Pitt, J. M.; André, F.; Amigorena, S.; Soria, J.-C.; Eggermont, A.; Kroemer, G.; Zitvogel, L. Dendritic cell-derived exosomes for cancer therapy. J. Clin. Investig 2016, 126, 1224-1232.

(33) Jang, S. C.; Kim, O. Y.; Yoon, C. M.; Choi, D.-S.; Roh, T.-Y.; Park, J.; Nilsson, J.; Lötvall, J.; Kim, Y.-K.; Gho, Y. S. Bioinspired exosome-mimetic nanovesicles for targeted delivery of chemotherapeutics to malignant tumors. ACS Nano 2013, 7, 7698.

(34) Goh, W. J.; Zou, S.; Ong, W. Y.; Torta, F.; Alexandra, A. F.; Schiffelers, R. M.; Storm, G.; Wang, J.-W.; Czarny, B.; Pastorin, G. Bioinspired Cell-Derived Nanovesicles versus Exosomes as Drug Delivery Systems: a Cost-Effective Alternative. Sci. Rep. 2017, 7, 14322.

(35) Antimisiaris, S. G.; Mourtas, S.; Marazioti, A. Exosomes and Exosome-Inspired Vesicles for Targeted Drug Delivery. Pharmaceutics 2018, 10, 218.

(36) Snell, A. A.; Neupane, K. R.; McCorkle, J. R.; Fu, X.; Moonschi, F. H.; Caudill, E. B.; Kolesar, J.; Richards, C. I. Cell-Derived Vesicles for in Vitro and in Vivo Targeted Therapeutic Delivery. ACS Omega 2019, 4, 12657-12664.

(37) Dong, X.; Gao, J.; Zhang, C. Y.; Hayworth, C.; Frank, M.; Wang, Z. Neutrophil Membrane-Derived Nanovesicles Alleviate Inflammation To Protect Mouse Brain Injury from Ischemic Stroke. ACS Nano 2019, 13, 1272-1283.

(38) Yurkin, S. T.; Wang, Z. Cell membrane-derived nanoparticles: emerging clinical opportunities for targeted drug delivery. Nanomedicine 2017, 12, 2007-2019.

(39) Doyle, L.; Wang, M. Overview of Extracellular Vesicles, Their Origin, Composition, Purpose, and Methods for Exosome Isolation and Analysis. Cells 2019, 8, 727.

(40) Choi, D.-S.; Kim, D.-K.; Kim, Y.-K.; Gho, Y. S. Proteomics of extracellular vesicles: Exosomes and ectosomes. Mass Spectrom. Rev. 2015, 34, 474-490.

(41) Kumar, A.; Dixit, C. K., 3 - Methods for characterization of nanoparticles; Elsevier Ltd, 2017; pp 43-58.

(42) Tan, X.; Zhang, Y.; Wang, Q.; Ren, T.; Gou, J.; Guo, W.; Yin, T.; He, H.; Zhang, Y.; Tang, X. Cell-penetrating peptide together with 
PEG-modified mesostructured silica nanoparticles promotes mucous permeation and oral delivery of therapeutic proteins and peptides. Biomater. Sci. 2019, 7, 2934-2950.

(43) Midekessa, G.; Godakumara, K.; Ord, J.; Viil, J.; Lättekivi, F.; Dissanayake, K.; Kopanchuk, S.; Rinken, A.; Andronowska, A.; Bhattacharjee, S.; Rinken, T.; Fazeli, A. Zeta potential of extracellular vesicles: toward understanding the attributes that determine colloidal stability. ACS Omega 2020, 5, 16701-16710.

(44) Gold, E. S.; Underhill, D. M.; Morrissette, N. S.; Guo, J.; McNiven, M. A.; Aderem, A. Dynamin 2 is required for phagocytosis in macrophages. J. Exp. Med. 1999, 190, 1849-1856.

(45) De Camilli, P.; Takei, K.; McPherson, P. S. The function of dynamin in endocytosis. Curr. Opin. Neurobiol. 1995, 5, 559-565.

(46) Sundborger, A. C.; Hinshaw, J. E. Regulating dynamin dynamics during endocytosis. F1000Prime Rep. 2014, 6, 85.

(47) Rappoport, J. Z.; Heyman, K. P.; Kemal, S.; Simon, S. M. Dynamics of Dynamin during Clathrin Mediated Endocytosis in PC12 Cells (Dynamin during Endocytosis). PLoS One 2008, 3, No. e2416.

(48) Feng, D.; Zhao, W.-L.; Ye, Y.-Y.; Bai, X.-C.; Liu, R.-Q.; Chang, L.-F.; Zhou, Q.; Sui, S.-F. Cellular internalization of exosomes occurs through phagocytosis. Traffic 2010, 11, 675-687.

(49) Kim, H.; Wang, S. Y.; Kwak, G.; Yang, Y.; Kwon, I. C.; Kim, S. H. Exosome-Guided Phenotypic Switch of M1 to M2 Macrophages for Cutaneous Wound Healing. Adv. Sci. 2019, 6, 1900513.

(50) Perry, V. H.; Teeling, J. Microglia and macrophages of the central nervous system: the contribution of microglia priming and systemic inflammation to chronic neurodegeneration. Semin. Immunopathol. 2013, 35, 601-612.

(51) Kanazawa, M.; Ninomiya, I.; Hatakeyama, M.; Takahashi, T.; Shimohata, T. Microglia and Monocytes/Macrophages Polarization Reveal Novel Therapeutic Mechanism against Stroke. Int. J. Mol. Sci. 2017, 18, 2135.

(52) Kigerl, K. A.; Gensel, J. C.; Ankeny, D. P.; Alexander, J. K.; Donnelly, D. J.; Popovich, P. G. Identification of two distinct macrophage subsets with divergent effects causing either neurotoxicity or regeneration in the injured mouse spinal cord. J. Neurosci. 2009, 29, 13435-13444.

(53) Park, K. M.; Bowers, W. J. Tumor necrosis factor-alpha mediated signaling in neuronal homeostasis and dysfunction. Cell. Signal. 2010, 22, 977-983.

(54) Bate, C.; Kempster, S. L.; Last, V.; Williams, A. Interferon- $\gamma$ increases neuronal death in response to amyloid- $\beta 1-42$. J. Neuroinflammation 2006, 3, 7.

(55) Conroy, S. M.; Nguyen, V.; Quina, L. A.; Blakely-Gonzales, P.; Ur, C.; Netzeband, J. G.; Prieto, A. L.; Gruol, D. L. Interleukin-6 produces neuronal loss in developing cerebellar granule neuron cultures. J. Neuroimmunol. 2004, 155, 43-54.

(56) Zhang, B.; Kopper, T. J.; Liu, X.; Cui, Z.; Van Lanen, S. G.; Gensel, J. C. Macrolide Derivatives Reduce Proinflammatory Macrophage Activation and Macrophage-Mediated Neurotoxicity. CNS Neurosci. Ther. 2019, 25, 591-600.

(57) Gensel, J. C.; Wang, Y.; Guan, Z.; Beckwith, K. A.; Braun, K. J.; Wei, P.; McTigue, D. M.; Popovich, P. G. Toll-Like Receptors and Dectin-1, a C-Type Lectin Receptor, Trigger Divergent Functions in CNS Macrophages. J. Neurosci. 2015, 35, 9966-9976.

(58) You, Q.; Gong, Q.; Han, Y. Q.; Pi, R.; Du, Y. J.; Dong, S. Z. Role of miR-124 in the regulation of retinoic acid-induced Neuro-2A cell differentiation. Neural Regen. Res. 2020, 15, 1133-1139.

(59) Sandin, J. N.; Aryal, S. P.; Wilkop, T.; Richards, C. I.; Grady, M. E. Near Simultaneous Laser Scanning Confocal and Atomic Force Microscopy (Conpokal) on Live Cells. JoVE 2020, 11, 162. 